\title{
Disconnected contributions to the spin of the nucleon
}

\author{
A. J. Chambers, ${ }^{1, *}$ R. Horsley, ${ }^{2}$ Y. Nakamura, ${ }^{3}$ H. Perlt, ${ }^{4}$ D. Pleiter, ${ }^{5,6}$ P. E. L. Rakow, ${ }^{7}$ G. Schierholz, ${ }^{8}$ \\ A. Schiller, ${ }^{4}$ H. Stüben, ${ }^{9}$ R. D. Young, ${ }^{1}$ and J. M. Zanotti ${ }^{1}$ \\ (CSSM and QCDSF/UKQCD Collaborations)
}

${ }^{1}$ CSSM, Department of Physics, University of Adelaide, Adelaide, South Australia 5005, Australia

${ }^{2}$ School of Physics and Astronomy, University of Edinburgh, Edinburgh EH9 3JZ, United Kingdom

${ }^{3}$ RIKEN Advanced Institute for Computational Science, Kobe, Hyogo 650-0047, Japan

${ }^{4}$ Institut für Theoretische Physik, Universität Leipzig, 04103 Leipzig, Germany

${ }^{5}$ JSC, Jülich Research Centre, 52425 Jülich, Germany

${ }^{6}$ Institut für Theoretische Physik, Universität Regensburg, 93040 Regensburg, Germany

${ }^{7}$ Theoretical Physics Division, Department of Mathematical Sciences, University of Liverpool, Liverpool L69 3BX, United Kingdom

${ }^{8}$ Deutsches Elektronen-Synchrotron DESY, 22603 Hamburg, Germany

${ }^{9}$ Regionales Rechenzentrum, Universität Hamburg, 20146 Hamburg, Germany

(Received 28 September 2015; published 30 December 2015)

\begin{abstract}
The spin decomposition of the proton is a long-standing topic of much interest in hadronic physics. Lattice QCD has had much success in calculating the connected contributions to the quark spin. However complete calculations, which necessarily involve gluonic and strange-quark contributions, still present some challenges. These "disconnected" contributions typically involve small signals hidden against large statistical backgrounds and rely on computationally intensive stochastic techniques. In this work we demonstrate how a Feynman-Hellmann approach may be used to calculate such quantities, by measuring shifts in the proton energy arising from modifications to the QCD action. We find a statistically significant nonzero result for the disconnected quark spin contribution to the proton of about $-5 \%$ at a pion mass of $470 \mathrm{MeV}$.
\end{abstract}

DOI: $10.1103 /$ PhysRevD.92.114517

PACS numbers: 12.38.Gc, 14.20.Dh

\section{INTRODUCTION}

The simple quark model picture suggests that the total nucleon spin is comprised entirely in terms of its constituent quark spins. In contrast, experimental measurements reveal that the quark spin only generates about one third of the total nucleon spin [1]. This observation is a clear representation of the nontrivial dynamics associated with nonperturbative QCD. Resolving the full composition of the nucleon spin in terms of the QCD degrees of freedom remains an active experimental and theoretical pursuit. For an overview of the status and progress, we refer the reader to Refs. [2-7].

As a systematically improvable method for studying nonperturbative properties of $\mathrm{QCD}$, lattice simulations offer the potential to provide quantitative predictions for the decomposition of the nucleon spin. For recent numerical investigations of the nucleon spin, and related matrix elements, see Refs. [8-16].

In the conventional approach, spin matrix elements are extracted from three-point correlation functions. Operator insertions that are directly connected to the quark field operators of the nucleon interpolators can be reliably

*alexander.chambers@adelaide.edu.au computed using established techniques. The operator insertions that involve self-contracted fermion lines, which are essential to isolate the strangeness spin content, for instance, require the stochastic estimation of the trace of an all-to-all propagator. Owing to the increased computational demand of this stochastic estimator and a relatively weak numerical signal, such disconnected contributions have often been neglected in lattice simulations. Nevertheless, substantial progress has been made in recent years [17-21]. For a related calculation involving the vector current matrix elements we also refer to Ref. [22].

In recent work, we have proposed an alternative to the conventional three-point function technique for the study of hadron matrix elements in lattice QCD. By adapting the Feynman-Hellmann (FH) theorem to the lattice framework, we are able to isolate matrix elements in terms of an energy shift in the presence of an appropriate weak external field $[23,24]$. This is similar to techniques proposed by e.g. [25-27]. In Ref. [23] we used the FH relation to extract the gluonic contribution to the nucleon mass. The application of FH was further developed in Ref. [24] for the study of the connected spin contributions in various hadrons. We have also recently shown how it is possible to compute flavor-singlet renormalization constants nonperturbatively by an appropriate application of the FH theorem [28]. 
In the present work, we apply the FH technique to resolve disconnected spin matrix elements. Whereas the connected spin contributions could be computed on conventional gauge fields, the disconnected contributions require the generation of new special-purpose gauge configurations. The influence of the weak external spin field is therefore accumulated through the importance sampling of the hybrid Monte Carlo simulation. While such new configurations come at significant computational cost, the computing time is comparable to that required for reliable sampling using the conventional stochastic techniques.

The manuscript proceeds as follows: Sec. II reviews the implementation of the FH theorem for the extraction of spin matrix elements and summarizes the lattice simulation parameters. Section III describes the strategy for the isolation of the relevant matrix elements from the twopoint correlation functions. Results are reported in Sec. IV, followed by concluding remarks in Sec. V.

\section{FEYNMAN-HELLMANN METHODS AND SIMULATION DETAILS}

Here we discuss the FH approach to calculations of disconnected contributions to matrix elements, in particular the quark axial charges. For details of previous calculations of the connected contributions, and the FH technique in general, see [24].

The quark axial charges are defined by forward matrix elements of the axial operator,

$$
\left\langle\vec{p}, \vec{s}\left|\bar{q}(0) \gamma_{\mu} \gamma_{5} q(0)\right| \vec{p}, \vec{s}\right\rangle=2 i s_{\mu} \Delta q .
$$

We access disconnected contributions to these quantities by implementing a modification to the fermion part of the QCD Lagrangian during gauge field generation. Extra terms are included which involve the axial operator weighted by a freely varying real parameter $\lambda$, applied equally to all three quark flavors:

$$
\mathcal{L} \rightarrow \mathcal{L}+\lambda \sum_{q=u, d, s} \bar{q} \gamma_{3} \gamma_{5} q
$$

This operator satisfies $\gamma_{5}$-Hermiticity, and so the functional determinant of the fermion matrix remains real. Hence we avoid introducing any sign problems. We choose projection matrices isolating spin-up and spin-down positive-parity components of the nucleon correlation function,

$$
\Gamma_{ \pm}=\frac{1}{2}\left(\mathbb{1}+\gamma_{4}\right) \frac{1}{2}\left(\mathbb{1} \pm i \gamma_{5} \gamma_{3}\right),
$$

and by application of the $\mathrm{FH}$ relation, find that the correlator picks up a complex phase which mimics an imaginary energy component,

$$
E \rightarrow E(\lambda)+i \phi(\lambda)
$$

At first order in $\lambda$, there is no shift in the real part of the energy, and the shift in the phase is exactly equal to the disconnected contribution to the total quark axial charge,

$$
\left.\frac{\partial E}{\partial \lambda}\right|_{\lambda=0}=0,\left.\quad \frac{\partial \phi}{\partial \lambda}\right|_{\lambda=0}= \pm \Delta \Sigma_{\text {disc }}
$$

The two signs for the phase shift result from the two different spin projections of $\Gamma_{ \pm}$, and we note that changing the spin projection is equivalent to flipping the sign of $\lambda . \Delta \Sigma_{\text {disc }}$ is the sum of individual flavor combinations,

$$
\Delta \Sigma_{\mathrm{disc}}=\Delta u_{\mathrm{disc}}+\Delta d_{\mathrm{disc}}+\Delta s
$$

where the strange contribution is purely disconnected, but we access the total quantity because the operator in Eq. (2) includes terms for all three simulated quark flavors.

Our strategy for the disconnected calculation, motivated by Eq. (5), is to generate new gauge ensembles for multiple values of $\lambda$, and determine $\Delta \Sigma_{\text {disc }}$ from the linear behavior of the phase shift in Eq. (4).

In our previous work, we were able to access the connected part of Eq. (1) by implementing the change in Eq. (2) to the Dirac matrix before inversion to compute the quark propagator entering hadron correlation functions (see [24]). Here the modification is made to the fermion matrix in the hybrid Monte Carlo algorithm, and so information about the purely disconnected contributions is accessed.

\section{Simulation details}

We use gauge field configurations with $2+1$ flavors of nonperturbatively $O(a)$-improved Wilson fermions and a lattice volume of $L^{3} \times T=32^{3} \times 64$. The lattice spacing $a=0.074(2) \mathrm{fm}$ is set using a number of singlet quantities [29-32]. The clover action used comprises the tree-level Symanzik improved gluon action together with a stout smeared fermion action, modified for the implementation of the FH method [24].

For the results discussed here, we use ensembles with two sets of hopping parameters, $\left(\kappa_{l}, \kappa_{s}\right)=(0.120900$, $0.120900)$ and $(0.121095,0.120512)$, corresponding to

TABLE I. Table of ensembles generated for this work. Two pion masses with three and two values of $\lambda$ respectively have been used. The number of configurations and sources used, as well as the phase shift measured (discussed in Sec. III and Sec. IV), are also listed.

\begin{tabular}{lcccc}
\hline \hline$\left(\kappa_{l}, \kappa_{s}\right)$ & $a \lambda$ & $N_{\text {conf }}$ & $N_{\text {sources }}$ & $\phi$ \\
\hline$(0.120900,0.120900)$ & -0.0125 & 500 & 1 & $0.00140(95)$ \\
& -0.00625 & 500 & 1 & $0.00002(83)$ \\
& 0.03 & 500 & 1 & $-0.00237(77)$ \\
$(0.121095,0.120512)$ & -0.025 & 600 & 1 & $-0.00076(130)$ \\
& 0.05 & 800 & 5 & $0.00027(61)$ \\
\hline \hline
\end{tabular}


pion masses of approximately 470 and $310 \mathrm{MeV}$. These have been generated with the modified quark action described in Eq. (2). The details of these ensembles, including the values of $\lambda$ realized, are given in Table I.

\section{ANALYSIS TECHNIQUES}

A standard zero-momentum projected nucleon correlation function is given by

$$
G_{ \pm}(t)=\int \mathrm{d}^{3} \vec{x} \Gamma_{ \pm}\langle\Omega|N(x) \bar{N}(0)| \Omega\rangle \stackrel{\text { large } t}{\longrightarrow} A e^{-E t},
$$

where $N$ and $\bar{N}$ are interpolating operators coupling to the nucleon ground state, and the projection matrices $\Gamma_{ \pm}$are defined in Eq. (3). For our simulations, we use identical source/sink smearing and operators, and hence the amplitude $A$ is purely real.

With the modification to the Lagrangian in Eq. (2), an imaginary component is introduced to the exponential factor in Eq. (7), in addition to a complex shift in the amplitude. This shift in the amplitude is not the focus of this work, but is related to the $\lambda$ dependence of the wave function overlap factors. To first order in $\lambda$, the amplitude and energy take the form

$$
\begin{gathered}
A \rightarrow A+\lambda(\Delta A+i \Delta B), \\
E \rightarrow E+i \lambda \Delta \Sigma,
\end{gathered}
$$

and the correlation function at large times is given by

$$
G_{ \pm}(\lambda, t) \stackrel{\text { large } t}{\longrightarrow}[A \pm \lambda(\Delta A+i \Delta B)] e^{-[E \pm i \lambda \Delta \Sigma] t},
$$

recalling that changing the spin projection is equivalent to changing the sign of $\lambda$. To extract the quantity $\lambda \Delta \Sigma$, we form a ratio of real and imaginary parts of spin-up and spin-down projections,

$$
\begin{aligned}
R(\lambda, t) & =\frac{\operatorname{Im}\left[G_{-}(\lambda, t)-G_{+}(\lambda, t)\right]}{\operatorname{Re}\left[G_{-}(\lambda, t)+G_{+}(\lambda, t)\right]} \\
& \stackrel{\text { large } t}{\longrightarrow} \frac{\sin (\lambda \Delta \Sigma t)-\lambda \frac{\Delta B}{A} \cos (\lambda \Delta \Sigma t)}{\cos (\lambda \Delta \Sigma t)+\lambda \frac{\Delta B}{A} \sin (\lambda \Delta \Sigma t)} .
\end{aligned}
$$

The large $t$ behavior of this ratio does not change if we include second order terms in Eq. (7) and Eq. (8). For the modification to the Lagrangian in Eq. (2), it can be shown that the second order shift in the nucleon energy is purely real, and corrections to Eq. (10) do not appear until $\mathcal{O}\left(\lambda^{3}\right)$.

The ratio in Eq. (10) is what we fit in our analysis. To determine ground state saturation of this quantity, we observe that, provided $t \ll \frac{1}{\mid \lambda \Delta \Sigma}$, the behavior of the ratio is approximately linear in $t$,

$$
R(\lambda, t)=\lambda \Delta \Sigma t-\lambda \frac{\Delta B}{A}, \quad a \ll t \ll \frac{1}{|\lambda \Delta \Sigma|} .
$$

Previous determinations of $\Delta \Sigma$ [17-21] suggest that we should expect $|\Delta \Sigma| \approx 0.1$, and hence for the largest value of $\lambda$ realized on our ensembles $(a \lambda=0.05)$, this linear approximation will hold for times $\frac{t}{a} \ll 200$. We introduce an "effective phase shift,"

$$
\phi_{\mathrm{eff}}=\frac{1}{a}[R(\lambda, t+a)-R(\lambda, t)]
$$

which in the regime discussed has the behavior

$$
\phi_{\mathrm{eff}}=\lambda \Delta \Sigma, \quad a \ll t \ll \frac{1}{|\lambda \Delta \Sigma|} .
$$

\section{RESULTS}

Figure 1 shows an example plot of the ratio in Eq. (10) and the corresponding effective phase defined in Eq. (13) for $a \lambda=0.03$. We observe a clear plateau in the effective phase for the illustrated fitting region, and corresponding linear behavior in the ratio. As an aside, the fit indicates a clearly nonzero value for the $t=0$ intercept, confirming that there is a small but statistically significant imaginary shift in the wave function overlap factors for this value of $\lambda$.

Extracting the phase shift for each value of $\lambda$, we calculate the linear dependence of the phase, shown in Fig. 2. From Eq. (5) we know that these linear shifts are directly proportional to $\Delta \Sigma_{\text {disc }}$. Since there is no phase shift in the zero-field limit, we have used a single-parameter slope fit to extract the linear term. This analysis is repeated
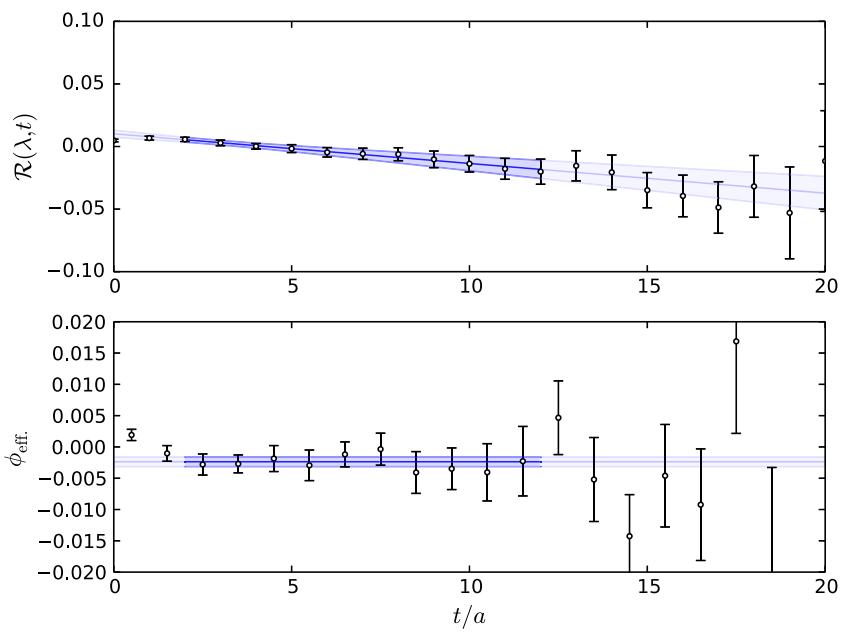

FIG. 1 (color online). Plots of the ratio in Eq. (10) and the effective phase shift defined in Eq. (13) for $a \lambda=0.03$, $m_{\pi} \approx 470 \mathrm{MeV}$. The fitting window (shown in darker blue) was between time slices 2 and 12. The errors shown are from a bootstrap analysis of the results, as are the errors on the displayed fits. 
A. J. CHAMBERS et al.

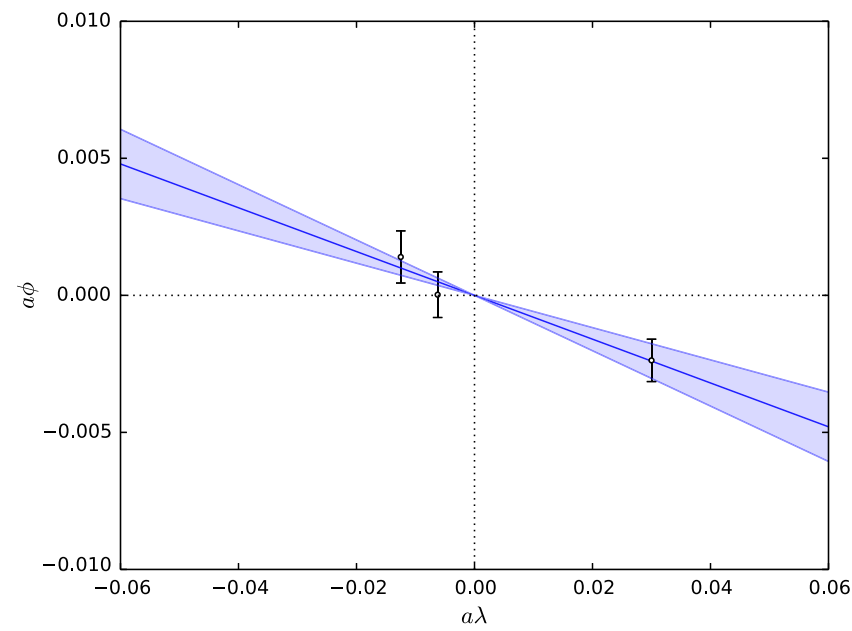

FIG. 2 (color online). Phase shift as a function of $\lambda$ for $m_{\pi} \approx 470 \mathrm{MeV}$.

at the lighter pion mass. Table I includes the calculated phase shift for each value of $\lambda$ on the ensembles generated, and results of the described analyses are summarized in Table II. Using the methods outlined in [24], we have also calculated the individual connected contributions to $\Delta \Sigma$ on these ensembles, and hence are able to calculate the total (connected and disconnected) value of $\Delta \Sigma$.

At the lighter mass, we find a result consistent with zero for $\Delta \Sigma_{\text {disc }}$. This unusual result may be the result of several different factors. The $\lambda$ values chosen may simply be too small, and the phase shift is not able to rise above the background correlator noise. Figure 3 shows effective phase plots for the two values of $\lambda$ realized at this lighter quark mass, and show that there is no clearly identifiable plateau at these statistics.

Alternatively, there may be a sign change in either the light or strange contribution to $\Delta \Sigma$ at some mass in the range $m_{\pi}=310-470 \mathrm{MeV}$. This is unlikely, however, as previous results at similar masses have shown a significant $\Delta s$ contribution, which would require the light quark contribution to have a strong quark mass dependence.

From [28] we have both nonsinglet and singlet renormalization factors for the axial current at the SU(3) symmetric point,

$$
Z_{A, \mathrm{NS}}^{\overline{\mathrm{MS}}(2 \mathrm{GeV})}=0.8458(8)
$$

PHYSICAL REVIEW D 92, 114517 (2015)
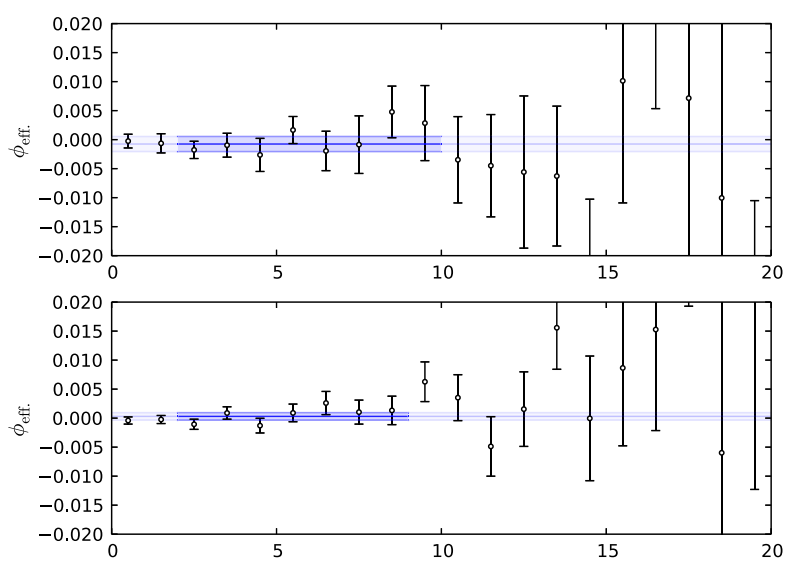

FIG. 3 (color online). Effective phase plots for $a \lambda=-0.025$, 0.05 respectively at $m_{\pi} \approx 310 \mathrm{MeV}$. The results in the second plot have greater statistics by a factor of 4 . Note that the sign of the fitted value is highly dependent on the fit window.

$$
Z_{A, \mathrm{~S}}^{\overline{\mathrm{MS}}(2 \mathrm{GeV})}=0.8662(34) .
$$

Further calculations at additional quark masses are required to perform a chiral extrapolation of these quantities; however the pion mass dependence of these factors is expected to be mild based on the nonsinglet calculation of Ref. [33].

To obtain the renormalized total spin contribution we use the singlet renormalization factor:

$$
\Delta \Sigma^{\overline{\mathrm{MS}}}=Z_{A, \mathrm{~S}}^{\overline{\mathrm{MS}}} \Delta \Sigma^{\mathrm{latt}}
$$

For the purely disconnected quantity, we include the mixing of the connected and disconnected contributions under renormalization:

$$
\Delta \Sigma_{\text {disc }}^{\overline{\mathrm{MS}}}=Z_{A, \mathrm{~S}}^{\overline{\mathrm{MS}}} \Delta \Sigma_{\mathrm{disc}}^{\mathrm{latt}}+\left(Z_{A, \mathrm{~S}}^{\overline{\mathrm{MS}}}-Z_{A, \mathrm{NS}}^{\overline{\mathrm{MS}}}\right) \Delta \Sigma_{\mathrm{conn}}^{\mathrm{latt}}
$$

Using the renormalization factors from the SU(3) symmetric point, we quote our $\overline{\mathrm{MS}}$ results in the final two columns of Table II.

Finally, since at the $\mathrm{SU}(3)$ symmetric point all quarks contribute exactly the same amount to $\Delta \Sigma_{\mathrm{disc}}^{\overline{\mathrm{MS}}}$, then at this point we can determine $\Delta s$ :

TABLE II. Table of results at each pion mass for the individual quark axial charges and the disconnected and full (connected plus disconnected) contribution to the total quark spin. The quantities reported with the "latt." superscript are unrenormalized. The final two columns report our renormalized results based on Eqs. (14) through (17).

\begin{tabular}{lccrrrr}
\hline \hline$\left(\kappa_{l}, \kappa_{s}\right)$ & $\Delta u^{\text {latt }}$ & \multicolumn{1}{c}{$\Delta d^{\text {latt }}$} & \multicolumn{1}{c}{$\Delta \Sigma_{\text {disc }}^{\text {latt }}$} & \multicolumn{1}{c}{$\Delta \Sigma^{\text {latt }}$} & \multicolumn{1}{c}{$\Delta \Sigma_{\text {disc }}^{\overline{\mathrm{MS}}(2 \mathrm{GeV})}$} & \multicolumn{1}{c}{$\Delta \Sigma^{\overline{\mathrm{MS}}(2 \mathrm{GeV})}$} \\
\hline$(0.120900,0.120900)$ & $1.001(7)$ & $-0.310(5)$ & $-0.079(21)$ & $0.612(24)$ & $-0.055(18)$ & $0.530(21)$ \\
$(0.121095,0.120512)$ & $1.004(10)$ & $-0.319(6)$ & $0.014(16)$ & $0.699(25)$ & $0.026(14)$ & $0.605(21)$ \\
\hline \hline
\end{tabular}


$\Delta s^{\overline{\mathrm{MS}}}\left(m_{\pi}=470 \mathrm{MeV}\right)=\frac{1}{3} \Delta \Sigma_{\mathrm{disc}}^{\overline{\mathrm{MS}}}=-0.018(6)$.

\section{CONCLUDING REMARKS}

Culminating in the results of Table II, we have shown how the FH theorem may be applied to perform full calculations of hadronic matrix elements.

Quantifying the computational cost of the FH approach relative to existing techniques is difficult, due to the wide variety of lattice and algorithmic schemes used. The calculation of $\Delta \Sigma_{\text {disc }}$ presented here at $m_{\pi} \approx 470 \mathrm{MeV}$ we estimate to be roughly equivalent to a total of 30,000 propagator calculations (measurements). This is based on the number of conjugate-gradient (CG) iterations performed during gauge field generation and Dirac matrix inversion. We estimate the results of [18] at a lighter pion mass of $m_{\pi} \approx 285$ required of the order of 100,000 measurements, based on the stated number of CG iterations, and an estimate of the number of inversions required for the calculation of a quark propagator at the simulated mass. Similarly the calculations in [20] are stated to have required approximately 150,000 measurements, at a pion mass of $370 \mathrm{MeV}$. All three techniques produce uncertainties which are broadly comparable, and hence we conclude that for this particular calculation, the FH method is at least competitive.

Extensions of the FH calculations include higherstatistics simulations, particularly at the lighter pion mass, and the generation of ensembles at additional pion masses to identify the quark mass dependence of $\Delta \Sigma$. Further analysis of the existing data should allow for the extraction of disconnected quark spin contributions for the other octet baryons and the vector mesons.

The FH technique demonstrated here can be easily adapted to a variety of other disconnected quantities, such as the gluonic contribution to angular momentum, which would otherwise be rather challenging using conventional approaches.

\section{ACKNOWLEDGMENTS}

The numerical configuration generation was performed using the BQCD lattice QCD program [34] on the IBM BlueGeneQ using DIRAC 2 resources (EPCC, Edinburgh, U.K.), the BlueGene P and Q at NIC (Jülich, Germany) and the Cray XC30 at HLRN (Berlin-Hannover, Germany). Some of the simulations were undertaken using resources awarded at the NCI National Facility in Canberra, Australia, and the iVEC facilities at the Pawsey Supercomputing Centre. These resources are provided through the National Computational Merit Allocation Scheme and the University of Adelaide Partner Share supported by the Australian Government. The BlueGene codes were optimized using Bagel [35]. The Chroma software library [36], was used in the data analysis. This investigation has been supported by the Australian Research Council under Grants No. FT120100821, No. FT100100005, and No. DP140103067 (R. D. Y. and J.M.Z.). H. P. was supported by DFG Grant No. SCHI 422/10-1.
[1] V. Yu. Alexakhin et al. (COMPASS Collaboration), Phys. Lett. B 647, 8 (2007).

[2] M. Anselmino, A. Efremov, and E. Leader, Phys. Rep. 261, 1 (1995); 281, 399(E) (1997).

[3] B. W. Filippone and X. Ji, Adv. Nucl. Phys. 26, 1 (2001).

[4] S. D. Bass, Rev. Mod. Phys. 77, 1257 (2005).

[5] F. Myhrer and A. W. Thomas, Phys. Lett. B 663, 302 (2008).

[6] A. W. Thomas, Phys. Rev. Lett. 101, 102003 (2008).

[7] C. A. Aidala, S. D. Bass, D. Hasch, and G. K. Mallot, Rev. Mod. Phys. 85, 655 (2013).

[8] J. D. Bratt et al. (LHPC Collaboration), Phys. Rev. D 82, 094502 (2010).

[9] S. N. Syritsyn et al., Proc. Sci., LATTICE2011 (2011) 178 [arXiv:1111.0718].

[10] S. Dinter, C. Alexandrou, M. Constantinou, V. Drach, K. Jansen, and D. B. Renner, Phys. Lett. B 704, 89 (2011).

[11] B. J. Owen, J. Dragos, W. Kamleh, D. B. Leinweber, M. Selim Mahbub, B. J. Menadue, and J. M. Zanotti, Phys. Lett. B 723, 217 (2013).
[12] S. Capitani, M. Della Morte, G. von Hippel, B. Jäger, A. Jüttner, B. Knippschild, H. B. Meyer, and H. Wittig, Phys. Rev. D 86, 074502 (2012).

[13] A. Sternbeck et al., Proc. Sci., LATTICE2011 (2011) 177 [arXiv:1203.6579].

[14] C. Alexandrou, M. Constantinou, S. Dinter, V. Drach, K. Jansen, C. Kallidonis, and G. Koutsou, Phys. Rev. D 88, 014509 (2013).

[15] T. Bhattacharya, S. D. Cohen, R. Gupta, A. Joseph, H.-W. Lin, and B. Yoon, Phys. Rev. D 89, 094502 (2014).

[16] G. S. Bali et al., Proc. Sci., LATTICE2013 (2014) 290 [arXiv:1311.7041].

[17] R. Babich, R. C. Brower, M. A. Clark, G. T. Fleming, J. C. Osborn, C. Rebbi, and D. Schaich, Phys. Rev. D 85, 054510 (2012).

[18] G. S. Bali et al. (QCDSF Collaboration), Phys. Rev. Lett. 108, 222001 (2012).

[19] M. Engelhardt, Phys. Rev. D 86, 114510 (2012).

[20] A. Abdel-Rehim, C. Alexandrou, M. Constantinou, V. Drach, K. Hadjiyiannakou, K. Jansen, G. Koutsou, and A. Vaquero, Phys. Rev. D 89, 034501 (2014). 
[21] M. Deka et al., Phys. Rev. D 91, 014505 (2015).

[22] J. Green, S. Meinel, M. Engelhardt, S. Krieg, J. Laeuchli, J. Negele, K. Orginos, A. Pochinsky, and S. Syritsyn, Phys. Rev. D 92, 031501 (2015).

[23] R. Horsley, R. Millo, Y. Nakamura, H. Perlt, D. Pleiter, P. E. L. Rakow, G. Schierholz, A. Schiller, F. Winter, and J. M. Zanotti (UKQCD, QCDSF Collaborations), Phys. Lett. B 714, 312 (2012).

[24] A. J. Chambers et al. (QCDSF/UKQCD, CSSM Collaborations), Phys. Rev. D 90, 014510 (2014).

[25] J. S. Schwinger, Phys. Rev. 82, 664 (1951).

[26] M. Foster and C. Michael (UKQCD Collaboration), Phys. Rev. D 59, 074503 (1999).

[27] W. Detmold, Phys. Rev. D 71, 054506 (2005).

[28] A. J. Chambers et al. (QCDSF Collaboration), Phys. Lett. B 740, 30 (2015).
[29] R. Horsley et al. (QCDSF-UKQCD Collaboration), Proc. Sci., LATTICE2013 (2013) 249 [arXiv:1311.5010].

[30] V. G. Bornyakov et al., arXiv:1508.05916.

[31] W. Bietenholz et al., Phys. Lett. B 690, 436 (2010).

[32] W. Bietenholz et al., Phys. Rev. D 84, 054509 (2011).

[33] M. Constantinou, R. Horsley, H. Panagopoulos, H. Perlt, P. E. L. Rakow, G. Schierholz, A. Schiller, and J. M. Zanotti, Phys. Rev. D 91, 014502 (2015).

[34] Y. Nakamura and H. Stüben, Proc. Sci., LATTICE2010 (2010) 040 [arXiv:1011.0199].

[35] P. A. Boyle, Comput. Phys. Commun. 180, 2739 (2009).

[36] R. G. Edwards and B. Joo (SciDAC, LHPC, UKQCD Collaborations), Nucl. Phys. B, Proc. Suppl. 140, 832 (2005). 